\title{
-Photogravure-
}

\section{Metronidazole-Induced Encephalopathy: A Case Report}

Eun Hui Bae ${ }^{1}$, Seong Kwon $\mathrm{Ma}^{1}$, Seul Kee Kim² and Soo Wan Kim ${ }^{1}$

${ }^{1}$ Department of Internal Medicine, Chonnam National University Medical School, Korea

${ }^{2}$ Department of Radiology, Chonnam National University Medical School, Korea
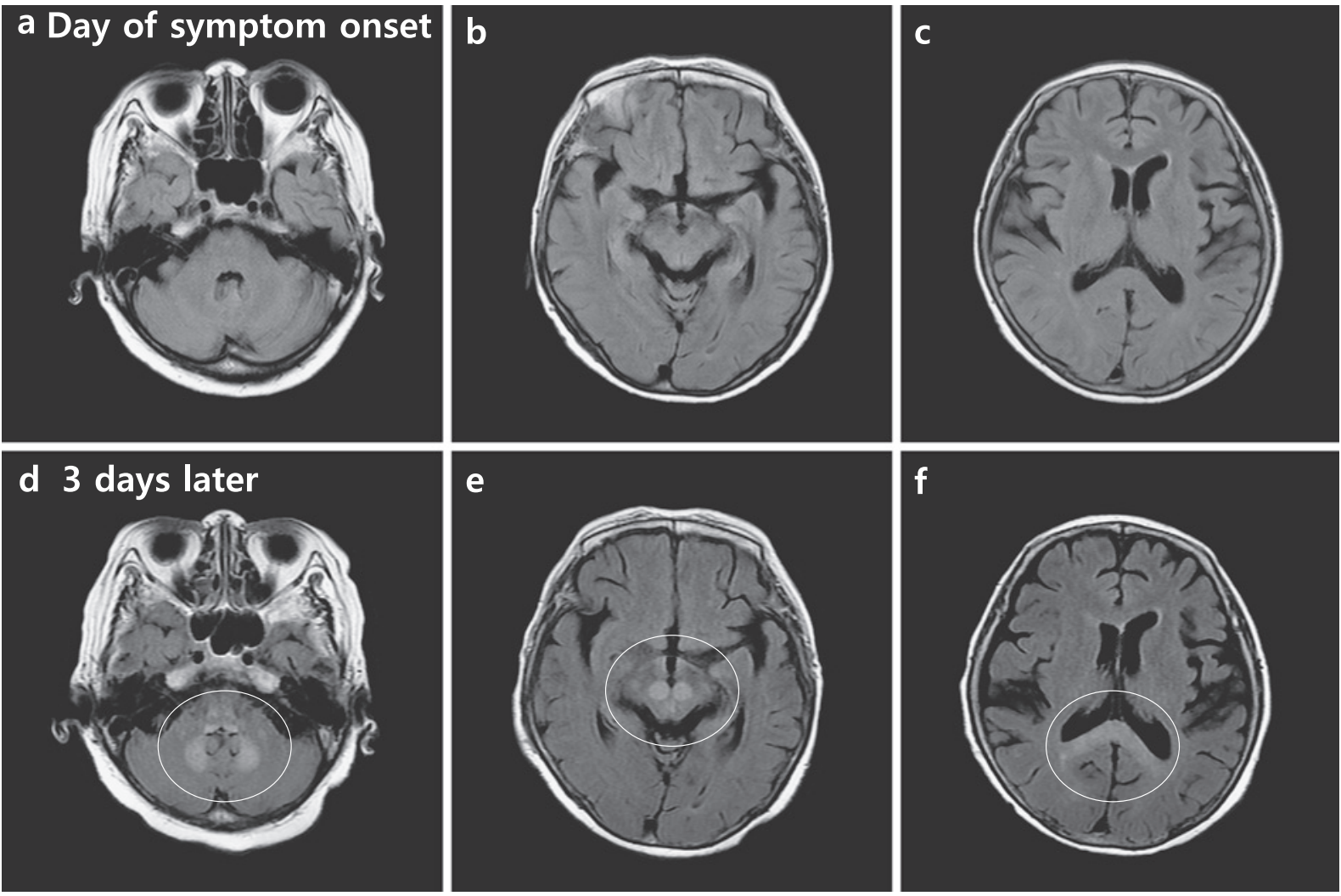

Fig. 1

Metronidazole-induced encephalopathy (MIE) is a rare clinical condition resulting from long-term use of the antimicrobial agent metronidazole. The symptoms of MIE typically include various forms of progressive neurological deterioration, such as gait disturbance, dysarthria, and dysmetria. These disturbances are accompanied by signal changes in the cerebellar dentate nuclei, corpus callosum, and midbrain upon neuroimaging. We report here the case of a 76-year-old woman underwent hemodialysis with MIE that was unusual due to the relatively short (7 days) duration of metronidazole treatment.

\section{Case Presentation}

A 76-year-old woman with end-stage renal disease, who had been on regular hemodialysis since 2009, was admitted with colitis and treated with metronidazole ( $1.5 \mathrm{~g} /$ day) for 7 days in a local hospital. After the 7th day, she was referred to our hospital's emergency room with ataxic gate and dysarthria, and admitted to the inpatient ward. Brain magnetic resonance imaging (MRI) was first performed on the day of her inpatient admission (i.e., symptom onset), and no abnormalities were detected (Fig. 1a-c). While an inpatient, she underwent regular hemodialysis with nutritional support for 3 days, but her neurological symptoms did not show any improve-

Correspondence to Soo Wan Kim, MD, PhD, Department of Internal Medicine, Chonnam National University Medical School, 42 Jebongro, Gwangju 501-757, Korea 
ment. We then performed another MRI, which showed hyperintense areas in the bilateral basal dentate nuclei (Fig. 1d), red nuclei (Fig. 1e), and splenium of the corpus callosum (Fig. 1f). Metronidazole-induced encephalopathy (MIE) was diagnosed, and the treatment with metronidazole of the patient was discontinued based on typical MRI findings. Without further intervention, her symptoms resolved completely on 2 days later after stopping metronidazole.

\section{Discussion}

This case demonstrates that short-term use of metronidazole can induce neurological symptoms of MIE, despite presenting with normal MRI signals in the early stage. Metronidazole is a widely used antimicrobial agent in clinical disorders including amebiasis, giardiasis, and certain anaerobic infections. Metronidazole can attains high plasma concentration in tissues, including the central nervous system, so it is the best drug for the treatment of anaerobic brain abscess ${ }^{1}$. Metronidazole is neurotoxic as a result of the oxidation of catecholamine neurotransmitters to semiquinone and nitro anion radicals, which reduce tissue oxygenation and subsequently generate superoxide radicals and hydrogen peroxide ${ }^{2}$. The total dose and duration of metronidazole producing MIE has been reported as $45 \mathrm{~g}-120 \mathrm{~g}$ and 1-12 weeks, respectively ${ }^{3-5}$. Notably, this patient was treated with a total of just 10.5 $\mathrm{g}$ metronidazole, and only for the minimal duration. Hemodialysis does not affect the clearance and metabolism of metronidazole ${ }^{6,7}$. Therefore, physicians should be aware of the possibility of metronidazole-induced encephalopathy at low cumulative doses, at least in end-stage renal disease patients, even if MRI findings do not indicate it.

Acknowledgements: This study was supported by a grant (CRI13903-21) from the Chonnam National University Hospital Biomedical Research Institute.

Conflict of Interest: There are no potential conflicts of interest affecting any authors of this paper.

Fig. 1 Brain magnetic resonance imaging (MRI) scans appeared normal even after gait disturbance developed $(\mathrm{a}-\mathrm{c})$. However, MRI 3 days after symptom development revealed hyperintense areas corresponding to the bilateral basal dentate nuclei (d), red nuclei (e), and corpus callosum splenium (f).

\section{References}

1. Lamp KC, Freeman CD, Klutman NE, Lacyt MK: Pharmacokinetics and pharmacodynamics of the nitroimidazole antimicrobials. Clin Pharmacokinet 1999; 36: 353-373.

2. Rao DN, Mason RP: Generation of nitro radical anions of some 5-nitrofurans, 2- and 5-nitroimidazoles by norepinephrine, dopamine, and serotonin. A possible mechanism for neurotoxicity caused by nitrohetrocyclic drugs. J Biol Chem 1987; 262: 11731-11736.

3. Ahmed A, Loes DJ, Bressler EL: Reversible magnetic resonance imaging findings in metronidazole-induced encephalopathy. Neurology 1995; 45: 588-589.

4. Bahn Y, Kim E, Park C, Park HC: Metronidazole induced encephalopathy in a patient with brain abscess. J Korean Neurosurg Soc 2010; 48: 301-304.

5. Lee SS, Cha SH, Lee SY, Song CJ: Reversible inferior colliculus lesion in metronidazole-induced encephalopathy: magnetic resonance findings on diffusion-weighted and fluid attenuated inversion recovery imaging. J Comput Assist Tomogr 2009; 33: 305-308.

6. Lau AH, Chang CW, Sabatini S: Hemodialysis clearance of metronidazole and its metabolites. Antimicrob Agents Chemother 1986; 29: 235-238.

7. Roux AF, Moirot E, Delhotal B, Leroy JA, Bonmarchand GP, Humbert G, Flouvat B: Metronidazole kinetics in patients with acute renal failure on dialysis: a cumulative study. Clin Pharmacol Ther 1984; 36: 363-368. 